\title{
Initiation and dynamics of a spiral wave around an ionic heterogeneity in a model for human cardiac tissue
}

\author{
Arne Defauw, ${ }^{1, *}$ Peter Dawyndt, ${ }^{2}$ and Alexander V. Panfilov ${ }^{1}$ \\ ${ }^{1}$ Department of Physics and Astronomy, Ghent University, Ghent, Belgium \\ ${ }^{2}$ Department of Applied Mathematics, Computer Science and Statistics, Ghent University, Ghent, Belgium \\ (Received 19 August 2013; revised manuscript received 18 October 2013; published 3 December 2013)
}

\begin{abstract}
In relation to cardiac arrhythmias, heterogeneity of cardiac tissue is one of the most important factors underlying the onset of spiral waves and determining their type. In this paper, we numerically model heterogeneity of realistic size and value and study formation and dynamics of spiral waves around such heterogeneity. We find that the only sustained pattern obtained is a single spiral wave anchored around the heterogeneity. Dynamics of an anchored spiral wave depend on the extent of heterogeneity, and for certain heterogeneity size, we find abrupt regional increase in the period of excitation occurring as a bifurcation. We study factors determining spatial distribution of excitation periods of anchored spiral waves and discuss consequences of such dynamics for cardiac arrhythmias and possibilities for experimental testings of our predictions.
\end{abstract}

DOI: 10.1103/PhysRevE.88.062703

PACS number(s): 87.19.Hh, 05.45.-a

\section{INTRODUCTION}

Contraction of the heart is initiated by the propagation of electrical waves of excitation. Electrical waves propagating through the heart belong to a large class of nonlinear waves that are widely studied theoretically in reaction-diffusion systems. One of the most important phenomena in such systems is the existence of vortices in the form of spiral waves of excitation. Spiral waves were found in a variety of nonlinear excitable media. In physicochemical systems, they have been observed in oscillating reactions [1,2] and heterogeneous catalysis $[3,4]$. Biological examples of such media include spiral waves of cAMP during morphogenesis of Dictyostelium discoideum amoebae [5,6], spiral waves of spreading depression in retina and in cortical tissue [7], calcium waves in Xenophus oocytes [8,9], and spiral waves in cardiac tissue [10-12]. In the heart, spiral waves underlie life threatening cardiac arrhytmias. One of the most important scientific questions for applications is to understand the mechanisms of initiation of spiral waves, i.e., of cardiac arrhythmias. Another important question is to find factors underlying their dynamics, as they are directly related to the type of cardiac arrhythmia [13,14]. It turns out that heterogeneity of cardiac tissue is important in the answer to both questions.

It was shown that heterogeneity substantially affects the dynamics of spiral waves. For example, spiral waves can drift because of heterogeneity [13-17]. Such drift can explain the onset of arrhythmia with periodically varying electrocardiogram (ECG), called torsades de pointes [16,18], or an arrhythmia with nonperiodic ECG: a polymorphic ventricular tachycardia [13,14].

Regarding the onset of spiral waves, it was shown that wave propagation at heterogeneities can be temporarily blocked $[19,20]$, and that such a pattern can evolve into spiral waves. The process of wave blocks and spiral wave formation in the presence of heterogeneity was studied in various modeling studies [21-26]. These studies showed that in order to be able to generate a 2D spiral wave, the heterogeneity should have

*Corresponding author: arne.defauw@ugent.be a substantial size, comparable to the wavelength of the spiral wave [21].

Most of the listed studies were performed using generic models of cardiac tissue and by using generic types of heterogeneity, as data on real heterogeneities, for example, in the human heart, were not available. Recently, measurements of heterogeneity in the human heart were performed [27]. Interestingly, in many cases the size of the heterogeneity was small. The possibility of formation of spirals, and the dynamics of spiral waves around such heterogeneities was not addressed, even at the generic level.

The aim of this paper is to study effects of heterogeneity of realistic size and value on the onset of spiral waves using the TP06 model [28] for human cardiac cells. We also study dynamics of spirals waves around such heterogeneities. In particular, we model heterogeneity similar to that measured by Glukhov in Ref. [27]. We apply high-frequency forcing and study if spiral waves can be formed in such a situation. We find that formation of spiral waves is possible. However, in all cases the created spiral wave will be anchored around the heterogeneity. Further, we study dynamics of such anchored spiral waves and factors determining its dynamics by varying the size and value of the heterogeneity. We discuss possible mechanisms of such dynamics and its importance for applications.

\section{MODELS AND METHODS}

Model. In this paper we consider a monodomain description of cardiac tissue [29], which has the following form:

$$
C_{m} \frac{\partial V_{m}}{\partial t}=\left(\frac{\partial}{\partial x_{i}} D_{i j} \frac{\partial V_{m}}{\partial x_{j}}\right)-I_{\text {ion }},
$$

where $D_{i j}$ is a diffusion matrix accounting for anisotropy of cardiac tissue, $i, j=1 \ldots n$, where $n=1$ in $1 \mathrm{D}, 2$ in $2 \mathrm{D} \ldots, C_{m}$ is membrane capacitance, $V_{m}$ is transmembrane voltage, $t$ is time, and $I_{\text {ion }}$ is the sum of ionic transmembrane currents describing the excitable behavior of individual ventricular cells. To represent human ventricular electrophysiological properties, we used the ionic TP06 model [28,30]. This model provides a detailed description of voltage, ionic currents, and 


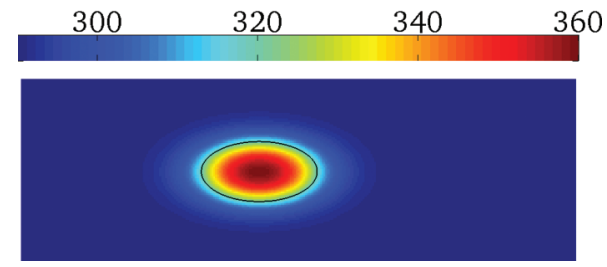

FIG. 1. (Color online) APD distribution in cardiac tissue simulated numerically in TP06 model [28,30]. Total size of the medium is $60 \mathrm{~mm} \times 20 \mathrm{~mm}$. Colormap shows APD in ms. Max. APD $=$ $359.5 \mathrm{~ms}$, min. APD $=290 \mathrm{~ms}$. Size at $50 \%$ heterogeneity is $11.2 \mathrm{~mm}$ on $5.6 \mathrm{~mm}$. Which is comparable to heterogeneity measured in the human heart [27]. In black, we show the size of the heterogeneity.

intracellular ion concentrations for human ventricular cells. A complete list of all equations can be found in Refs. [28,30]. We used the default parameter settings from Ref. [28] for epicardial cells. All parameter changes made to obtain tissue heterogeneity are detailed in the text.

Numerical methods. For 1D and 2D computations, the forward Euler method was applied to integrate Eq. (1). A space step of $\Delta x=0.2 \mathrm{~mm}$ and a time step of $\Delta t=0.02 \mathrm{~ms}$ were used. To integrate the Hodgkin-Huxley-type equations for the gating variables of the various time-dependent currents $\left(m, h\right.$, and $j$ for $I_{\mathrm{Na}} ; r$ and $s$ for $I_{\mathrm{to}} ; x_{r 1}$ and $x_{r 2}$ for $I_{\mathrm{Kr}} ; x_{s}$ for $I_{\mathrm{Ks}} ; d, f, f_{2}$, and $f_{\mathrm{Cass}}$ for $\left.I_{\mathrm{CaL}}\right)$, the Rush and Larsen scheme [31] was used.

Anisotropy. In most of our simulations, the fibers are directed along the $x$ axis. In few simulations we study effect of rotational anisotropy, in that case the diffusion matrix is given by

$$
\begin{aligned}
& D_{x x}=D_{L} \cos ^{2} \theta+D_{T} \sin ^{2} \theta, \\
& D_{x y}=D_{y x}=0, \\
& D_{y y}=D_{T},
\end{aligned}
$$

with $\theta(y)=\frac{y}{d}\left(\theta_{2}-\theta_{1}\right)+\theta_{1}$. Here $d$ is the distance between epicardium and endocardium, $\theta_{1}=-60^{\circ}, \theta_{2}=60^{\circ}, D_{L}=$ $0.128 \frac{\mathrm{mm}^{2}}{\mathrm{~ms}}$, and $D_{T}=D_{L} / 4$.

Heterogeneity. To study heterogeneity, we change the parameters $G_{\mathrm{Ks}}, G_{\mathrm{Kr}}$, and $G_{\mathrm{CaL}}$ from their default values 0.392 $\mathrm{nS} / \mathrm{pF}, 0.153 \mathrm{nS} / \mathrm{pF}$, and $3.980 \times 10^{-5} \frac{\mathrm{cm}}{\mathrm{ms} \mu \mathrm{F}}$ for epicardial

(a)

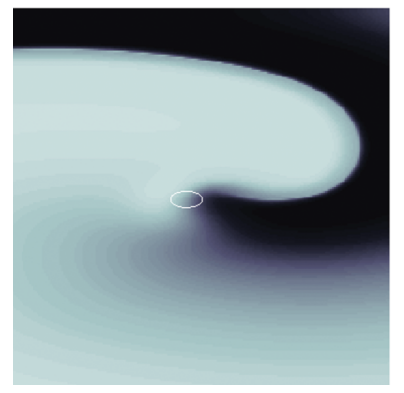

(b)

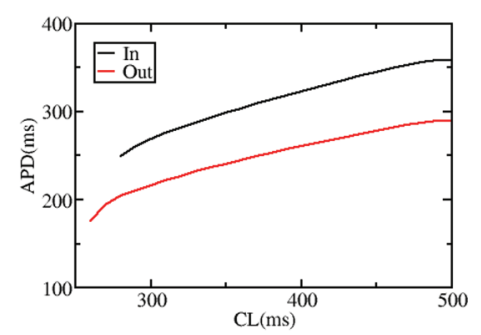

FIG. 2. (Color online) (a) A spiral wave in homogeneous epicardial tissue. White lines shows the tip trajectory. Total size of the medium is $40 \mathrm{~mm} \times 40 \mathrm{~mm}$. (b) Dynamic APD restitution curve. In black the restitution curve for a cell located at the center of the heterogeneity shown in Fig. 1. In red the restitution curve for a cell located outside the heterogeneity.

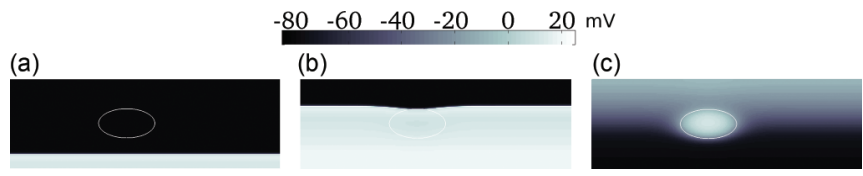

FIG. 3. (Color online) Wave propagation at pacing rate $T=$ $380 \mathrm{~ms}$. White line shows size of the heterogeneity. Time interval between (a) and (b) is $40 \mathrm{~ms}$, between (b) and (c) it is $240 \mathrm{~ms}$.

cells in Ref. [28]. Typical profile of heterogeneity is shown in Fig. 1. Spiral wave dynamics for homogeneous cardiac tissue is shown in Fig. 2(a). We see that it has a circular core and shows stationary rotation. Figure 2(b) shows dynamic APD restitution curve for the homogeneous tissue (red) and inside the heterogeneous tissue configuration used as a base line model (black). We see that inside the heterogeneity the restitution curve has more shallow slope (maximal slope of 0.9 for the black line compared to 1.1 for the red line).

Electrocardiogram. To calculate the ECG, we used the formula for the potential from [16]: $\Phi=\sum \partial V / \partial x_{i} \partial / \partial x_{i}\left(\frac{1}{R}\right)$, where $\sum$ denotes the summation over all points of the numerical grid, $i=1,2$ is the index for the coordinate axes and $R$ is the distance from a lead to the point of the heart where $\partial V / \partial x_{i}$ is evaluated.

\section{RESULTS}

\section{A. Baseline model}

Figure 1 shows our baseline tissue configuration. It is qualitatively similar to heterogeneity of the human ventricular tissue measured in Ref. [27]. In particular, the maximal and minimal values of APD are approximately the same and the size at $50 \%$ heterogeneity in both cases is around $10 \mathrm{~mm} \times 6 \mathrm{~mm}$. The exact underlying reason of the APD difference in Ref. [27] was not studied. However, as for the case of other APD heterogeneities between cardiac cells studied experimentally in Refs. [32,33] it can be achieved by changing $I_{\mathrm{Kr}}$ and $I_{\mathrm{Ks}}$ conductances. In our case, we did it by setting $G_{\mathrm{Ks}}=0.3751 \mathrm{nS} / \mathrm{pF}, G_{\mathrm{Kr}}=0.1532 \mathrm{nS} / \mathrm{pF}$ outside the heterogeneity, and $G_{\mathrm{Ks}}=0 \mathrm{nS} / \mathrm{pF}, G_{\mathrm{Kr}}=0.0948 \mathrm{nS} / \mathrm{pF}$ inside the heterogeneity. These values were initially estimated using an approach we developed earlier [34].

In the first series of simulations, we studied behavior of waves around the heterogeneity at high-frequency pacing. We paced the medium from below at increasingly faster pacing rates. We started with a pacing rate $T=400 \mathrm{~ms}$. After 10 stimuli, this pacing rate was decreased with $10 \mathrm{~ms}$, and so on. We observed the following changes in the wave patterns (see movies in Supplemental Material [35]). For relatively slow pacing rate (period $T>290 \mathrm{~ms}$ ), the effect of heterogeneity on wavefront is small [Figs. 3(a) and 3(b)] and its only influence is longer repolarization time [Fig. 3(c)]. For frequencies in the interval $T<290 \mathrm{~ms}$, we see the formation of two breaks [Fig. 4(a)], which penetrate into the heterogeneity from above [Figs. 4(b) and 4(c)] as in classical mechanisms for spiral wave initiation [19,21]. However, the size of the heterogeneity is too small, and there is not enough room for the onset of spiral waves via this mechanism. If frequency becomes faster than $270 \mathrm{~ms}$, the wave cannot penetrate the heterogeneity. So we get a classical Wenckebach 1:2 block at the heterogeneity (see 


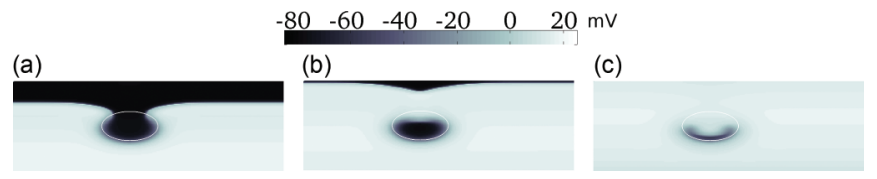

FIG. 4. (Color online) (a) Formation of two breaks at pacing rate $T=280 \mathrm{~ms}$. (b) and (c) The breaks enter the heterogeneity from above. Time interval between the frames is $20 \mathrm{~ms}$.

Fig. 5). Again, no spirals are formed. Thus, we conclude that due to small size of the heterogeneity, we do not observe spiral wave formation at the heterogeneity via a classical mechanism as in Ref. [19].

However, if we further increase the frequency of stimulation, we find that the effect of heterogeneity on waves spreads to the boundary of the medium (Fig. 6). In particular, at some stage, stimulation of the medium produces a wave consisting of two disjoint wavebreaks [Fig. 6(a)]. Further increase in frequency of stimulation increases the gap between the wavebreaks [Fig. 6(c)]. Because the heterogeneity was not centrally located, such increasing gap eventually results in the disappearance of the left break. Interaction of the right break with the heterogeneity eventually leads to a clockwise rotating spiral wave [Fig. 7(c)]. After this, the picture becomes selfreproducing: a rotating wave interacts with external forcing and reproduces itself after each next stimulation. If we stop external stimulation at this stage, we get a single spiral wave rotating around and anchored to the heterogeneity. We note that the direction of rotation of the spiral wave is not necessarily the same as for Fig. 7(c); see also Sec. IIID. It depends on the interaction of the right break with the heterogeneity, on the moment of time when we stop external forcing and on the recovery pattern of the tissue around the heterogeneity.

We performed several simulations following this protocol and we always found, after we stopped the stimulation, either disappearance of spiral waves or a single spiral wave rotating around the heterogeneity. We conclude that at heterogeneities similar to those measured by Glukhov in Ref. [27], a spiral source can be generated and these spiral sources will be anchored to these heterogeneities. Our next step is to study the dynamics of such anchored excitation source.

Figure 8 shows typical dynamics for a spiral wave rotating around the heterogeneity. The first figure $(t=0 \mathrm{~ms})$ shows such phase of rotation when the heterogeneity is in the refractory state. The spiral rotates around it as around an inexcitable obstacle (0-200 ms). However, after some time, the refractory state at the heterogeneity ends and it becomes excitable again. Now the spiral can enter this region (around time $=220 \mathrm{~ms}$ ). Subsequently, the wave will exit the heterogeneity and will

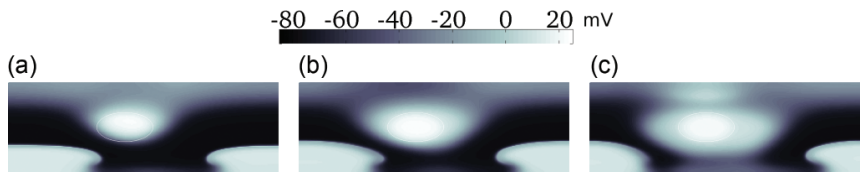

FIG. 6. (Color online) Wave propagation at pacing rate $T=240$ (a) and $T=230$ (b) and (c). Time interval between (a) and (b) is 480 $\mathrm{ms}$; between (b) and (c), $460 \mathrm{~ms}$.

join with the wavefront of the spiral wave (time $=260 \mathrm{~ms}$ ). After this, the process is repeated.

\section{B. Periods}

Let us characterize the process of rotation of a spiral wave in these conditions, i.e., for an anchored spiral wave around a heterogeneity, as in Fig. 8. In Fig. 9 we show the period of excitation in each point of the medium.

We see two distinct values for the period: $T_{2}=360 \mathrm{~ms}$ at and around the heterogeneity and $T_{1}=240 \mathrm{~ms}$ in other parts of the tissue. We also see that the longer period region mainly coincides with the heterogeneity. Let us study the factors that determine these two values of the period.

We first change $G_{\mathrm{Ks}}$ and $G_{\mathrm{Kr}}$ outside the heterogeneity, keeping the same values inside it. As in the previous case, we generated a spiral wave rotating and anchored around the heterogeneity and found period of excitation in the medium. Figure 10(a) shows the values of the period inside $\left(T_{2}\right)$ and outside $\left(T_{1}\right)$ the heterogeneity versus APD outside the heterogeneity. We observe a gradual increase of $T_{1}$ with increase of APD. We also see a slight increase of the period inside the heterogeneity. This is due to the effect of the surrounding tissue on the heterogeneity properties: value of APD inside heterogeneity slightly increases when APD outside is increased, because of electronic effects [36].

We also altered $G_{\mathrm{Ks}}$ and $G_{\mathrm{Kr}}$ inside the heterogeneity, keeping the same values outside. The results are shown in Figs. 10(b) and 11. We see that the period outside the heterogeneity $\left(T_{1}\right)$ is almost unchanged. The period inside the heterogeneity $\left(T_{2}\right)$ is severely affected by this change. For a small heterogeneity [Figs. 11(a)-11(d)], we do not observe two clear distinct values of the period. Spatially in this case, we have a large region around the heterogeneity with slightly increased period. For large heterogeneity, $\mathrm{APD}_{\max }>340$ [Figs. 11(e)-11(k)], we have a typical two-period distribution similar to that of Fig. 9. We also observe a clear bifurcation: an abrupt change in $T_{2}$ around $\mathrm{APD}_{\max } \approx 340$ [Fig. 10(b)].

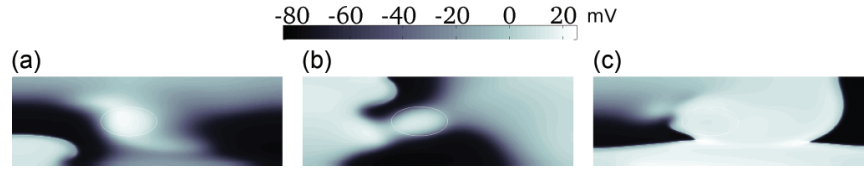

FIG. 7. (Color online) Wave propagation at pacing rate $T=220$. (a) Single break formation; (b), (c) Formation of a single spiral wave rotating around and anchored to the heterogeneity. Time interval between (a) and (b) is $320 \mathrm{~ms} ; 120 \mathrm{~ms}$ between (b) and (c).
FIG. 5. (Color online) Wave propagation for pacing rate $T=$ $260 \mathrm{~ms}$ : the breaks cannot penetrate the heterogeneity. We get a classical Wenckebach 1:2 block at the heterogeneity. Time interval between the frames is $40 \mathrm{~ms}$.

(c)

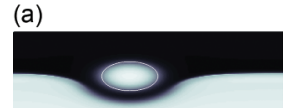

(b)

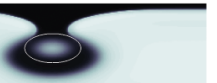

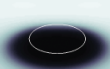



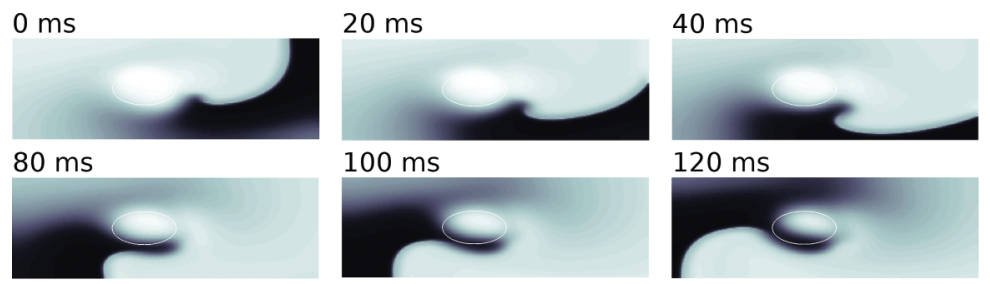

$120 \mathrm{~ms}$
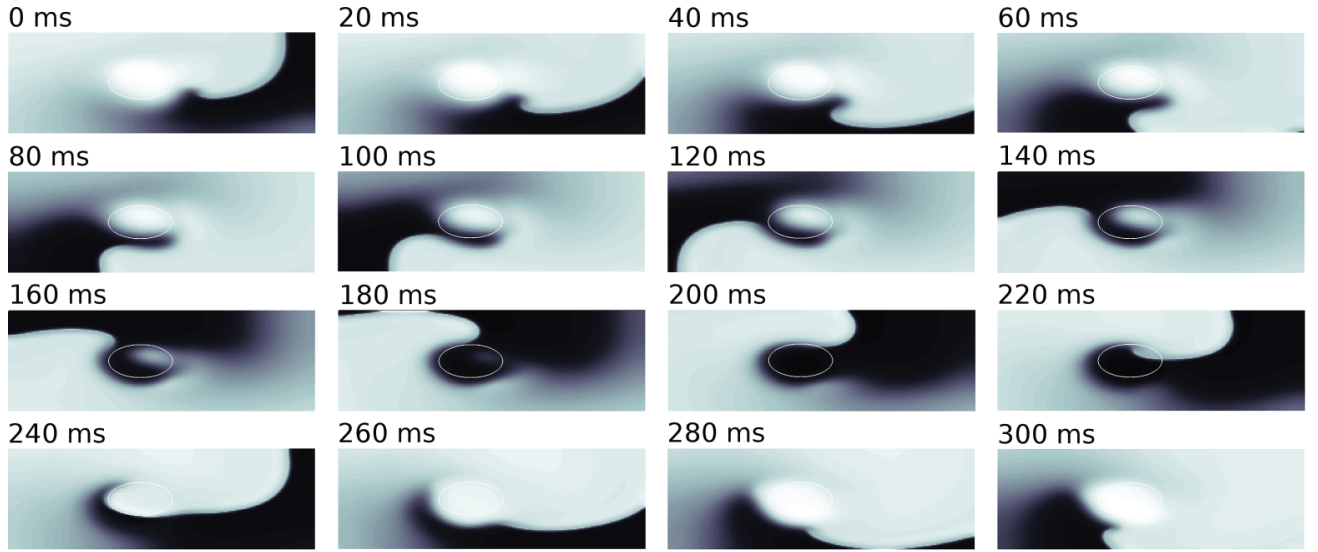

$260 \mathrm{~ms}$
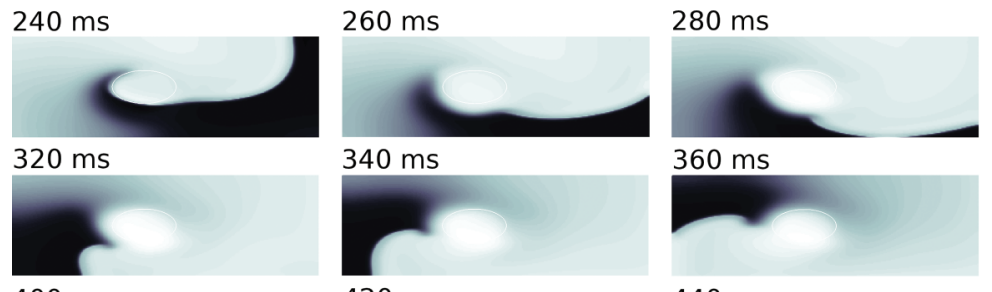

$400 \mathrm{~ms}$

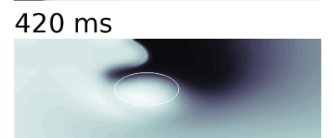

$360 \mathrm{~ms}$

$300 \mathrm{~ms}$
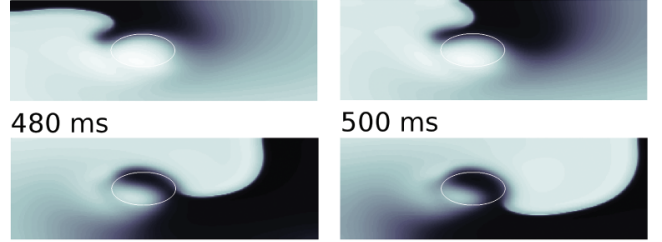

$560 \mathrm{~ms}$

$580 \mathrm{~ms}$
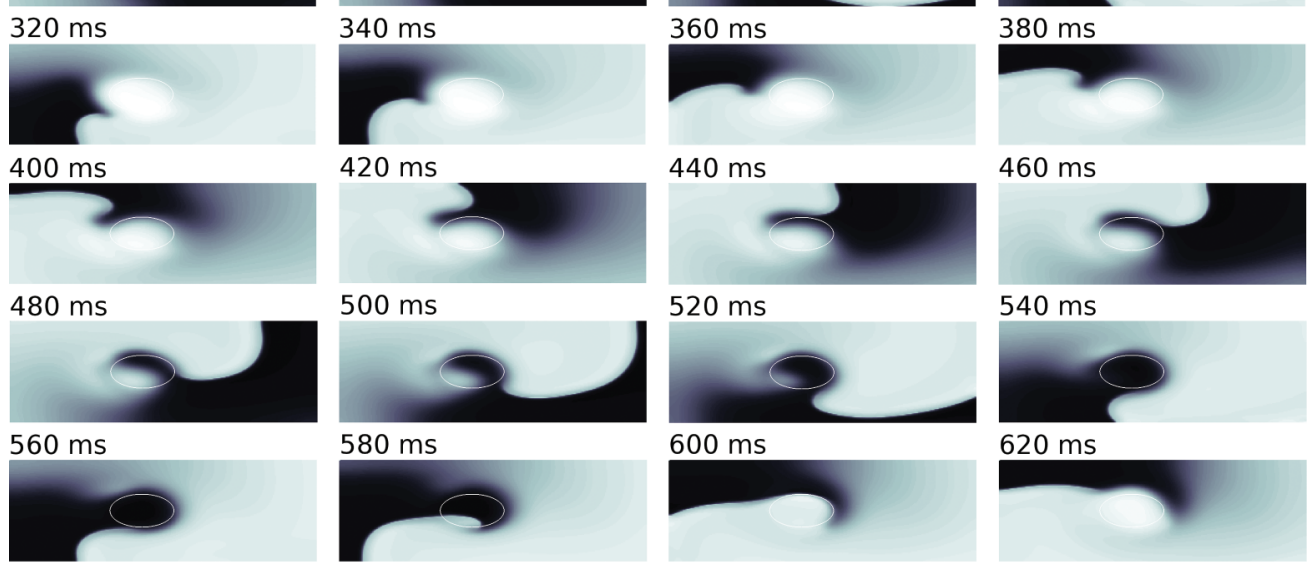

$520 \mathrm{~ms}$

$640 \mathrm{~ms}$
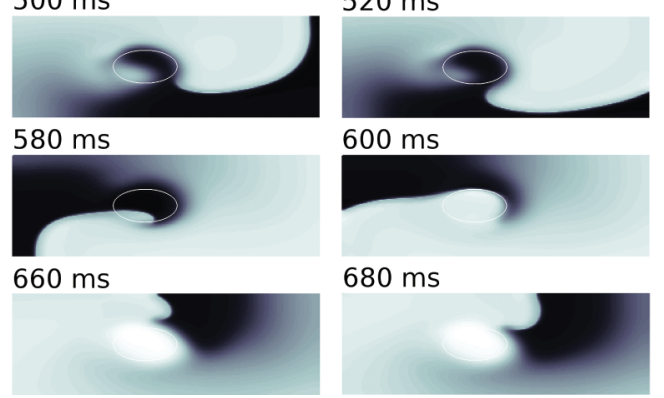

$600 \mathrm{~ms}$

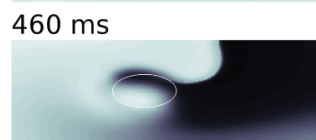

$540 \mathrm{~ms}$
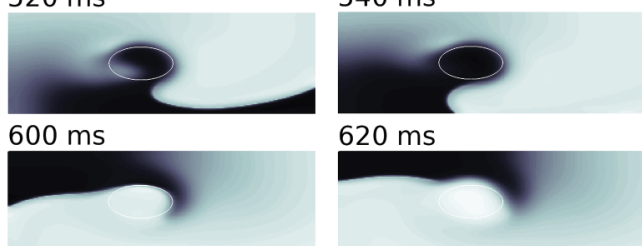

$680 \mathrm{~ms}$

$620 \mathrm{~ms}$
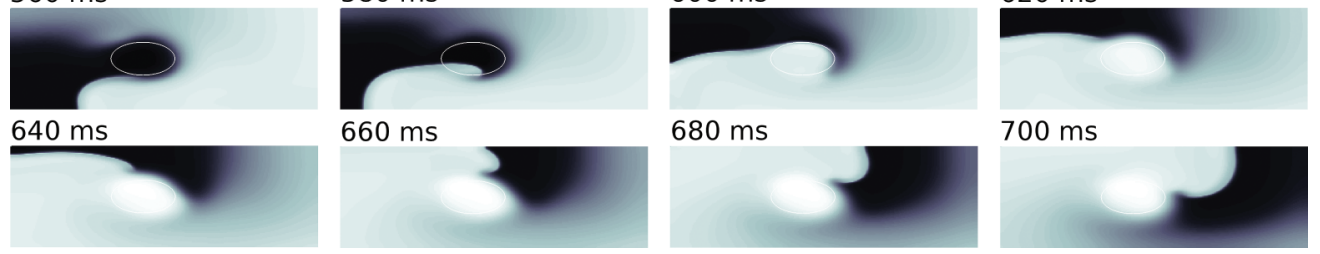

$700 \mathrm{~ms}$

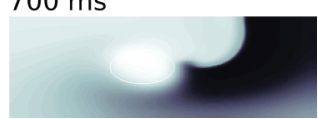

FIG. 8. (Color online) Rotation of spiral wave anchored around heterogeneity of Fig. 1. Figures show wave pattern at 20-ms intervals. White line shows size of the heterogeneity.

\section{Period increase bifurcation}

Bifurcation in the period of excitation obviously results in a change of the wave propagation pattern. If we consider two successive points where the spiral wave tip enters the heterogeneity, we observe the following dynamics before and after the period jump. For smaller heterogeneity [Fig. 11(a)], the wave tip makes a rotation of about 380 degrees before entering the heterogeneity again. To show it in Figs. 11(a)11(d), we marked by black and red arrows the entry points of the wave into the heterogeneity for two successive rotations (first black, then red). For larger heterogeneity the rotation is

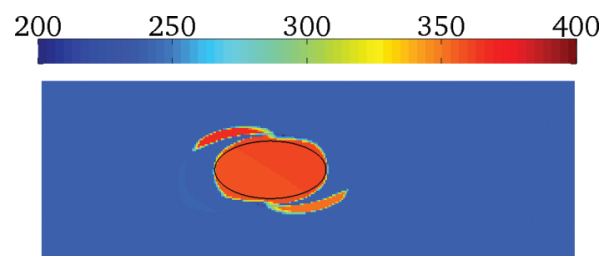

FIG. 9. (Color online) Period of excitation of the medium for spiral wave dynamics shown in Fig. 8. Figure shows average value of period in each point over 15 excitations. Period inside the heterogeneity $\left(T_{2}\right)$ is approximately $360 \mathrm{~ms}$. Period in the other part of the medium $\left(T_{1}\right)$ is around $240 \mathrm{~ms}$. about 390 degrees [Fig. 11(b)]. Thus, rotation angle increases when we increase heterogeneity. However, at the bifurcation point, it approaches 400 degrees and then it jumps to about 540 degrees, which results in an abrupt period increase.

We also found that this bifurcation only occurs in a limited range of size of the heterogeneity. Figure 12 shows results similar to Fig. 10(b), but now with different sizes of the heterogeneity: we increased the size, respectively, 1.2, 1.5, 1.7, and 2 times. We see that in Figs. 12(a)-12(c), we have qualitatively the same bifurcation as in Fig. 8(b).However, the (a)

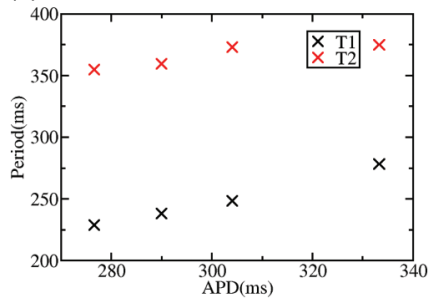

(b)

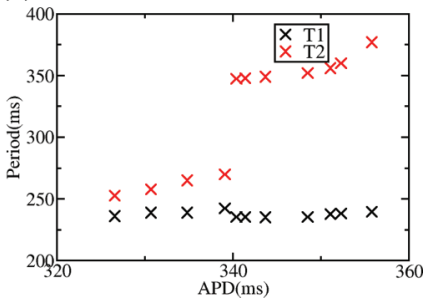

FIG. 10. (Color online) (a) $T_{1}$ and $T_{2}$ versus APD outside the heterogeneity: we alter $G_{\mathrm{Ks}}$ and $G_{\mathrm{Kr}}$ outside the heterogeneity. (b) $T_{1}$ and $T_{2}$ versus the maximal value of APD inside the heterogeneity: we alter $G_{\mathrm{Ks}}$ and $G_{\mathrm{Kr}}$ inside the heterogeneity. 
(a)

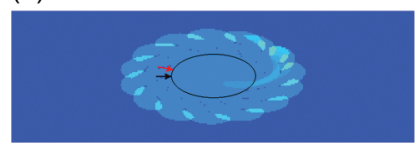

(c)

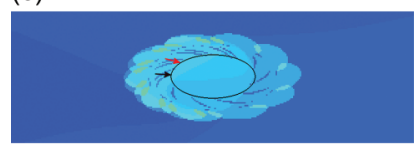

(e)

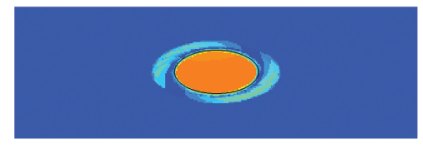

(g)

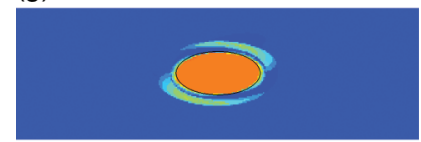

(i)

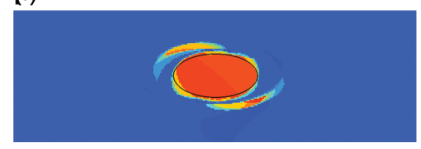

\section{(b)}

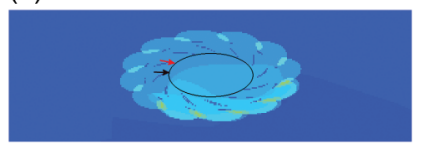

(d)

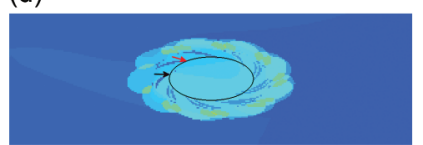

(f)

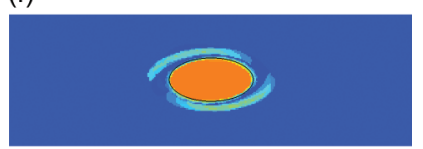

(h)

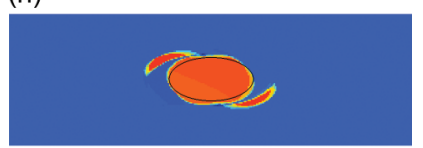

(j)

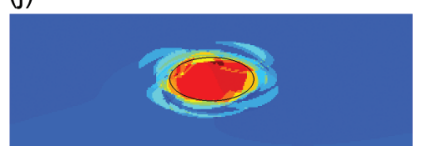

FIG. 11. (Color online) Period of excitation of the medium for spiral wave dynamics anchored around a heterogeneity with different maximal APD values. The ionic properties of the cells inside the heterogeneity were changed, resulting in different maximal APD values. The size of the heterogeneity is kept constant and the same as described in the legend of Fig. 1. Total size of the medium is $60 \mathrm{~mm} \times 20 \mathrm{~mm}$. Outside the heterogeneity we have in all cases: $G_{\mathrm{Ks}}=0.3751 \mathrm{nS} / \mathrm{pF}, G_{\mathrm{Kr}}=0.1532 \mathrm{nS} / \mathrm{pF}$. Parameter values inside the heterogeneity were in: (a) $G_{\mathrm{Ks}}=0.1226 \mathrm{nS} / \mathrm{pF}, G_{\mathrm{Kr}}=$ $0.1532 \mathrm{nS} / \mathrm{pF}$; (b) $G_{\mathrm{Ks}}=0.0981 \mathrm{nS} / \mathrm{pF}, G_{\mathrm{Kr}}=0.1532 \mathrm{nS} / \mathrm{pF}$; (c) $G_{\mathrm{Ks}}=0.0736 \mathrm{nS} / \mathrm{pF}, G_{\mathrm{Kr}}=0.1532 \mathrm{nS} / \mathrm{pF}$; (d) $G_{\mathrm{Ks}}=$ $0.049 \mathrm{nS} / \mathrm{pF}, G_{\mathrm{Kr}}=0.1532 \mathrm{nS} / \mathrm{pF}$; (e) $G_{\mathrm{Ks}}=0.0421 \mathrm{nS} / \mathrm{pF}, G_{\mathrm{Kr}}=$ $0.1532 \mathrm{nS} / \mathrm{pF}$; (f) $G_{\mathrm{Ks}}=0.0368 \mathrm{nS} / \mathrm{pF}, G_{\mathrm{Kr}}=0.1532 \mathrm{nS} / \mathrm{pF}$; (g) $G_{\mathrm{Ks}}=0.0245 \mathrm{nS} / \mathrm{pF}, G_{\mathrm{Kr}}=0.1532 \mathrm{nS} / \mathrm{pF}$; (h) $G_{\mathrm{Ks}}=0 \mathrm{nS} / \mathrm{pF}$, $G_{\mathrm{Kr}}=0.1149 \mathrm{nS} / \mathrm{pF}$; (i) $G_{\mathrm{Ks}}=0 \mathrm{nS} / \mathrm{pF}, G_{\mathrm{Kr}}=0.0948 \mathrm{nS} / \mathrm{pF}$; (j) $G_{\mathrm{Ks}}=0 \mathrm{nS} / \mathrm{pF}, G_{\mathrm{Kr}}=0.0479 \mathrm{nS} / \mathrm{pF}$.

location of the bifurcation on the APD axis slightly increases with the size of the heterogeneity. The amplitude of the jump first also slightly increases [Figs. 10(b), 12(a), and 12(b)], then decreases [Fig. 12(c)], and finally the bifurcation disappears [Fig. 12(d)].

Note that this bifurcation in period has a substantial effect on overall dynamics of the system. Figure 13 shows how it is manifested on the ECG. We see that even tiny changes in the extent of the heterogeneity results in ECGs of very different type: ECG with gradual amplitude variation as in Fig. 13(a),which is reminiscent of torsades de points (before the bifurcation point) and ECG with large beat-tobeat variations in amplitude (after the bifurcation). We also see substantial shift in the secondary peaks in the Fourier transforms of ECG before and after the bifurcation, which indicates the recorded change in $T_{2}$.

(a)

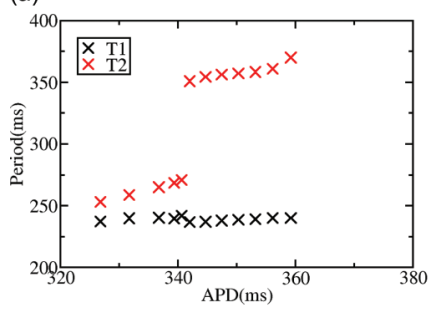

(b)

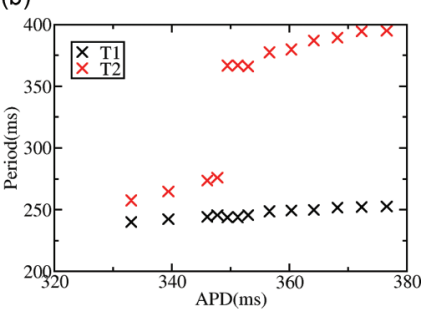

(c)

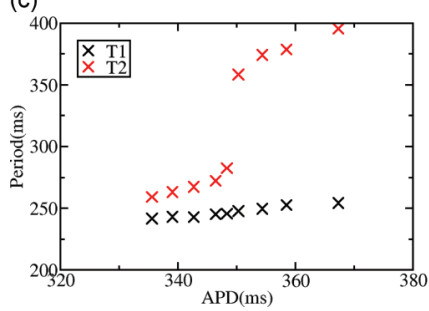

(d)

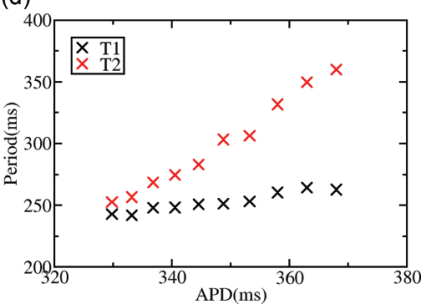

FIG. 12. (Color online) $T_{1}$ and $T_{2}$ versus the maximal value of APD inside the heterogeneity for different sizes of the heterogeneity. In (a), the axes of the heterogeneity are 1.2 times larger than in the basic model; (b)-(d), axes are 1.5, 1.7, and 2 times larger, respectively.

\section{Modifications of baseline model}

So far, we have studied the process of spiral wave onset and period increase bifurcation for one particular shape of heterogeneity, anisotropy, and parameter set. Here we extend our study to additional configurations.

In Fig. 1, heterogeneity in APD was created by changing $G_{\mathrm{Ks}}$ and $G_{\mathrm{Kr}}$ conductances. A similar heterogeneity can be created by changing other parameter values. For instance, another current that has a substantial effect on APD is $I_{\mathrm{CaL}}$. We have checked if our results also hold for heterogeneity created by changing $G_{\mathrm{CaL}}$ instead of $G_{\mathrm{Kr}}$. In particular, we studied a heterogeneity of the same elliptical shape as in our baseline model and used inside the value $G_{\mathrm{CaL}}=6.766 \times 10^{-5} \frac{\mathrm{cm}}{\mathrm{ms} \mu \mathrm{F}}$ (an increase by a factor 1.7) and $G_{\mathrm{Ks}}=0 \mathrm{nS} / \mathrm{pF}$. Outside the

(a)

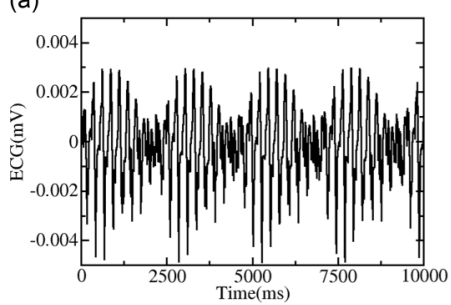

(c)

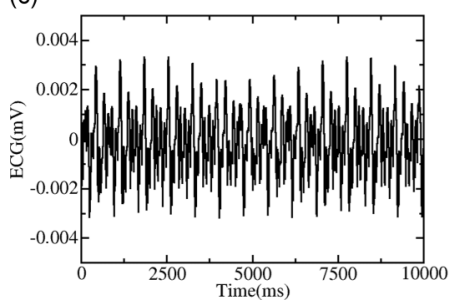

(b)

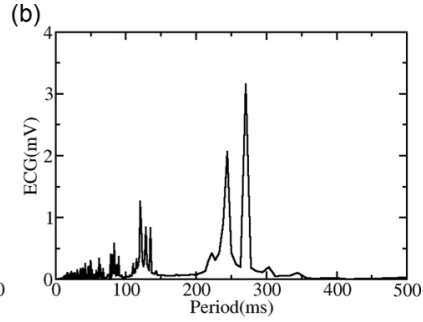

(d)

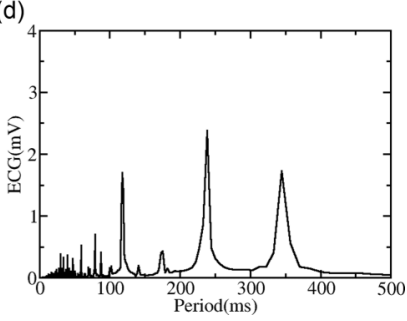

FIG. 13. ECG and corresponding Fourier-transform. (a) ECG for the heterogeneity as in Fig. 11(d). (b) Fourier-transform of this ECG profile. We see two dominant periods corresponding to $T_{1}$ and $T_{2}$. (c), (d) ECG and Fourier-transform for heterogeneity as in Fig. 11(e). Again, we find the two dominant periods $T_{1}$ and $T_{2}$. 


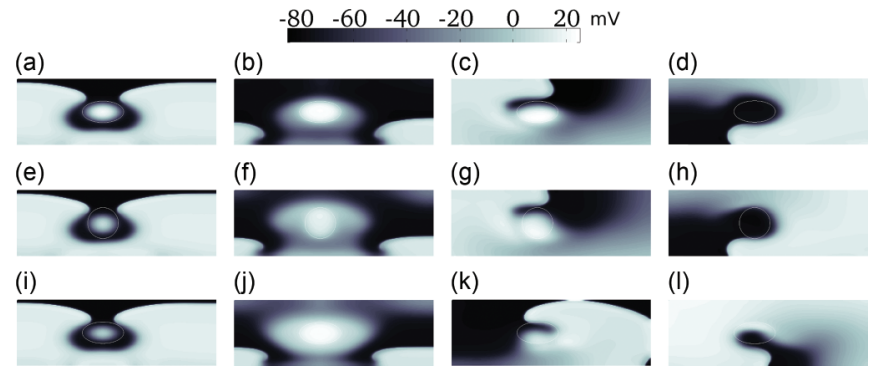

FIG. 14. (Color online) Spiral wave initiation under highfrequency pacing. Upper panel: heterogeneity is created by changing $G_{\mathrm{CaL}}=6.766 \times 10^{-5} \frac{\mathrm{cm}}{\mathrm{ms} \mu \mathrm{F}}$ and $G_{\mathrm{Ks}}=0 \mathrm{nS} / \mathrm{pF}$. (a) Formation of wavebreaks $(T=260 \mathrm{~ms})$. (b) Wavebreak dynamics under higher pacing rate $(T=250 \mathrm{~ms})$. (c), (d) Formation of a single spiral wave rotating around and anchored to the heterogeneity. Middle panel: simulations for a circular heterogeneity with radius $4.5 \mathrm{~mm}$. Parameter values inside and outside the heterogeneity are the same as for the baseline model. (e) $T=260 \mathrm{~ms}$; (f) $T=240 \mathrm{~ms}$. (g), (h) Spiral wave anchored to the heterogeneity. Lower panel: similar simulations for model with rotational anisotropy (details are in the text). Parameter values inside and outside the heterogeneity are the same as for the baseline model. (i) $T=260 \mathrm{~ms}$; (j) $T=240 \mathrm{~ms}$. (k), (1) Spiral wave anchored to the heterogeneity.

heterogeneity, we used the same parameter values as in our baseline model. This results in a difference between maximal and minimal APD of $62 \mathrm{~ms}$, which is comparable to the heterogeneity shown in Fig. 1.

We performed the same simulations as we did for our baseline model. First, we studied behavior of waves around the heterogeneity at high frequency pacing. The results are shown in the Supplemental Material movies [35] and in Fig. 14 (upper panel). In Fig. 14(a), we see that we get the formation of wavebreaks, which cannot penetrate the heterogeneity, as in Fig. 5. Again, if we further increase pacing rate, we observe that the size of the heterogeneity increases: the effect of heterogeneity spreads to the boundary of the medium [see Fig. 14(b)]. Similar to Fig. 7 it results in complex patterns of excitation, and eventually in the formation of a single clockwise rotating spiral wave anchored to the heterogeneity (see Figs. 14(c) and 14(d)].

Next, we did the same analysis for the period of excitation, for the spiral wave anchored to the heterogeneity, as for the baseline model. We found similar results: two distinct values of period for different parts of the tissue $\left(T_{2} \approx 370 \mathrm{~ms}\right.$ inside the heterogeneity, $T_{1} \approx 240 \mathrm{~ms}$ outside the heterogeneity). We also studied if we have a similar bifurcation as for the baseline model. For that, we varied $G_{\mathrm{Ks}}$ inside the heterogeneity, and measured the period of excitation. The results are shown in Fig. 15(a). We observe a clear bifurcation, similar to that of Fig. 10(b).

In the second series of simulations, we changed the shape of the heterogeneity from an ellipse to a circle that has approximately the same area. We used the same parameter values inside and outside the heterogeneity as for the baseline model, resulting in the same difference between maximal and minimal APD as in Fig. 1. We also studied behavior under high frequency pacing: see supplementary movie [35] and Fig. 14 (middle panel). Again, we get the formation of a single clockwise rotating anchored spiral, created as a result of similar processes [compare Figs. 14(a)-14(d) with Figs. 14(e)-14(h)].

We found similar results for period of excitation: two clear distinct values $\left(T_{2} \approx 360 \mathrm{~ms}\right.$ and $\left.T_{1} \approx 240 \mathrm{~ms}\right)$ for other parts of the tissue. We also varied $G_{\mathrm{Ks}}$ and $G_{\mathrm{Kr}}$ inside the heterogeneity [Fig. 15(b)]. Again, we observe two regimes indicating the bifurcation.

Finally, we studied possible effect of rotational anisotropy on our results. The reason for this is that the measurement of heterogeneity in Ref. [27] were performed in a transmural wedge of the left ventricular free wall. It is known that the direction of the fibers is not constant along a transmural wedge: the fibers rotate counterclockwise from endocardium to epicardium. At the endocardium, the angle between the fibers and the $x-y$ plane is around $-60^{\circ}$; at the epicardium it is around $60^{\circ}$ [37]. Therefore, we studied if such fiber rotation might have an effect on our results. We refer to the method section for the diffusivity matrix under rotational anisotropy. The distance $d$ between epicardium and endocardium was in our case $20 \mathrm{~mm}$. Again, we performed the same simulations as in previous situations. We used the same elliptical shape of the heterogeneity as in our baseline model, and the same parameter values inside and outside the heterogeneity, as in Fig. 1. This results in a difference between maximal and minimal APD of $62 \mathrm{~ms}$. We refer to the Supplemental Material movies [35] and lower panel of Fig. 14 for the results under high frequency pacing. We observe similar behavior as in our other situations. Although, the direction of the resulting spiral wave is now opposite to previous simulations.

For the period of excitation we also find two clear distinct values ( $T_{2} \approx 360 \mathrm{~ms}$ and $T_{1} \approx 240 \mathrm{~ms}$ ) for different parts of the tissue. By changing $G_{\mathrm{Ks}}$ and $G_{\mathrm{Kr}}$ inside the heterogeneity, (a)

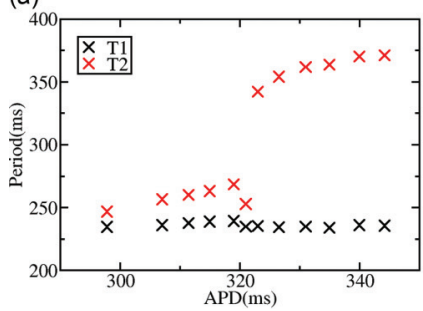

(b)

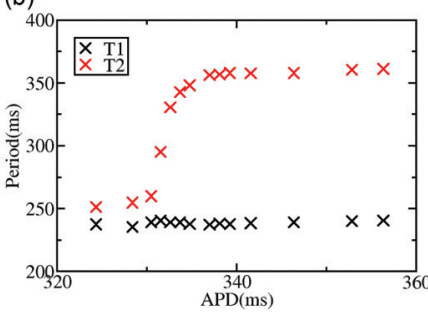

(c)

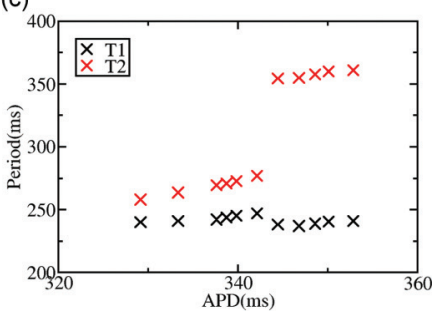

FIG. 15. (Color online) $T_{1}$ and $T_{2}$ versus the maximal value of APD inside the heterogeneity. A: Simulations in which APD inside the heterogeneity was changed by changing $G_{\mathrm{Ks}}$ at $G_{\mathrm{CaL}}=6.766 \times 10^{-5} \frac{\mathrm{cm}}{\mathrm{ms} \mu \mathrm{F}}$; (b) and (c): Same simulations as for the baseline model in Fig. 10(b), but now with a circular shape of the heterogeneity, respectively rotational anisotropy. 
we find a similar bifurcation pattern as in Fig. 10(b) [see Fig. 15(c)].

\section{DISCUSSION}

In this paper, we study possible effects of small-sized heterogeneities, similar to those found in human cardiac tissue, on initiation of spiral waves and their dynamics. We found that new spiral waves can be formed even on a small heterogeneity with a size around $1 \mathrm{~cm}$. However, initiation of these new sources involves not only break formation as in classical mechanisms [19,21] but also interaction of wavebreaks with other upcoming waves, which eventually results in the formation of a single spiral wave anchored around the heterogeneity.

Previous studies of the TP06 model [28] show that slopes of the restitution curves, corresponding to parameter values used here, do not result in the onset of dynamical instabilities leading to breakup. We have also performed a simulation in a medium without heterogeneity and did not observe any dynamical instabilities there.

In our simulations spirals are initiated by the complex interaction between the wavebreaks and the heterogeneity. In particular, the heterogeneity in our simulations was not located in the center of the tissue. This brakes the symmetry of our model. Because of a successive increase in distance between the generated wavebreaks, one of the wavebreaks eventually disappears at the boundary, leading to the formation of a spiral wave. Thus, formation of a single spiral wave here does depend on initial asymmetry, which, however, is likely to be present in any realistic setup.

We studied the dynamics of these anchored spiral waves. We found that in such systems we have two distinct values of period of excitation: one inside and one outside the heterogeneity. We show that each of these periods is mainly determined by properties of cardiac tissue at the corresponding region: increase of the refractory period results in increase of the period. The ratio of these periods is not given by an integer number. At first glance, this contradicts the classic view of excitation of heterogeneity by Wenckebach [38], who showed that at fast pacing rate, block of excitation at the heterogeneity results in an integer ratio in period of excitation inside and outside of the heterogeneity. Note, however, that in our case, the wave enters the heterogeneity at different places, which results in the existence of noninteger ratios of periods of excitation. Similar effect in 3D was found in Ref. [39], where it was shown that in a 3D heterogeneous medium, the ratio is not given by an integer number.

We found that increase in period inside the heterogeneity, when we increase the extent of heterogeneity, is not gradual: we find a bifurcation point. At this bifurcation point, the period suddenly increases around 1.3 times.

The mechanism of this bifurcation is still under investigation. It is not trivial and involves interplay of several factors, such as heterogeneity size, heterogeneity value, and relation of the refractory periods and the rotation time of the wave around the heterogeneity. In a very simplified way, the jump in period can be explained in the following way. The heterogeneity can be excited when the refractory period inside the heterogeneity ends. However, the refractory period (a)

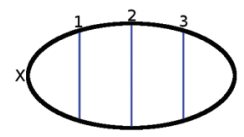

(b)

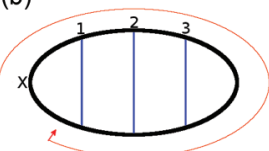

(c)

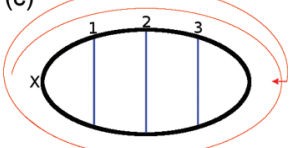

FIG. 16. (Color online) Schematic explanation of mechanism behind the bifurcation. (a) Wave propagation through the heterogeneity after it enters it at point X. (b) Rotation of a wave tip around the heterogeneity. The wave follows the boundary of the heterogeneity along the red arrow. The wave enters the heterogeneity again in the region around point (c): Same as (b), but for a larger heterogeneity. The wave enters the heterogeneity after making an extra half rotation in comparison to (b), because of increased heterogeneity.

depends on the history of wave propagation through the heterogeneity, as is schematically shown in Fig. 16(a).

If the wave enters the heterogeneity at point $X$, its front will have successive positions marked as line 1, 2, and 3. Wave propagation will thus have an effect on the spatial distribution of the recovery time. Indeed, the refractory period will first end at point $\mathrm{X}$, and then at lines 1,2 , and 3 . This is because points at line 1 were excited later than at point $X$ and thus it will recover at a later time. Let us now consider rotation of a wave around such heterogeneity and assume that the tip of the spiral wave first enters the heterogeneity at point $\mathrm{X}$. It will follow the boundary of the heterogeneity as shown by a red arrow in Fig. 12(b) and the wave will be able to enter again when the tissue at the tip location is recovered. For a heterogeneity with a longer refractory period this will take a longer time. The tip will thus make a rotation for an increasingly larger angle, as we saw in Fig. 11, which results in a gradual increase of the period $T_{2}$. However, if the value of the refractory period at the heterogeneity grows, the wave, even after coming back to the region around point $\mathrm{X}$, will not be able to enter the heterogeneity, as the tissue is still not recovered there. In that case, the tip has to travel further heading to line 1 . However, at line 1 the tissue will recover later than at point $X$ and the wave will not be able to enter the heterogeneity there as well. The reason for this is, as discussed above, that the wave tip propagates along the same trajectory as the wave that made the heterogeneity refractory at the previous excitation. This effect will disappear after making an additional half rotation [see Fig. 16(c)].

Such simple schematic consideration can explain the onset of bifurcation in Fig. 10(b) and even predict that after the bifurcation the tip of the spiral has to make 1.5 rotation around the heterogeneity before entering it. However, this consideration is, in many aspects, oversimplified: it does not take into account many other important effects, for example, possible difference in velocity of the wave inside the heterogeneity and the wave tip outside the heterogeneity. It also does not consider the effects of wavefront curvature etc. As a result, in reality the wave can make a rotation slightly more than 360 degrees (the bifurcation occurs at a rotation angle of around 400 degrees). Also, the jump is slightly less than 1.5 rotation (in reality it is 1.44 ). Finally, such simple consideration cannot explain disappearance of the bifurcation for larger sizes of the heterogeneity as shown in Fig. 12(d). For a heterogeneity of larger size, the pattern of rotation of the wave around the heterogeneity changes: instead of rotating 
around it, the wave tip periodically propagates through the heterogeneous region and is not anchored to it anymore, as we saw in Fig. 8.

We checked that our results on the dynamics of spiral wave around the heterogeneity are general and hold in a medium of different size, different anisotropy, different shape, and for the heterogeneities induced by modification of different parameters of our model.

Experimental studies that are somewhat similar to situations studied in our paper were performed in Ref. [40]. In that paper, the authors studied wave dynamics in the presence of a heterogeneity of $0.8 \mathrm{~cm} \times 0.8 \mathrm{~cm}$ in a rabbit heart. After giving several premature stimuli, they observed that wavebreaks were formed at the place where the heterogeneity was located. After further pacing, they observed the formation of two counterrotating waves of which only one survives, resulting in a single spiral wave rotating around the heterogeneity, as in our study. Unfortunately, the authors did not measure spatial distribution of period of excitation. However, dynamics look close to what we observed, as the authors saw breakthrough in the heterogeneity, as well as the rotation of a spiral wave around it.

Compared to other modeling studies [16,18,21], the main effects of heterogeneity on spiral wave dynamics in our study is its anchoring effect and not drift along the heterogeneity boundary. We also get a torsades de pointes-like ECG and an ECG reminiscent of polymorphic tachycardia. However, in our case it is a result of a different frequency of excitation of tissue inside and outside the heterogeneity and not a result of shift of the excitation source in space.

Note that anchoring of spiral waves in 2D and scroll waves in $3 \mathrm{D}$ was a subject of intensive study [12,41-43]. However, in all these cases the spiral waves were anchored around inexcitable regions in $2 \mathrm{D}$ or $3 \mathrm{D}$. Here we show that a heterogeneity that is excitable can also anchor spirals. Compared to anchoring around an inexcitable obstacle, anchoring here results in more complex dynamics because of a direct influence of the heterogeneity on wave rotation.

Dynamics of waves in the presence of large nonconducting and ionic heterogeneities was studied in Ref. [44]. It was shown that dynamics of waves, including anchoring, is dependent on the location of the heterogeneity in the tissue.

Finding the bifurcation described in our paper in an experimental study might not be easy, as it requires a gradual change of the extent of heterogeneity, which is difficult to obtain in experiments at the whole organ level. However, it might be possible to use cell cultures of neonatal rat ventricular myocytes, such as in Refs. [45,46], where heterogeneities of various form and size can be created. Further gradual changing of the extent of the heterogeneity can be achieved by application of drugs changing the refractory period of cardiac tissue.

\section{ACKNOWLEDGMENTS}

A.D., P.D., and A.V.P. acknowledge the support of Ghent University (Multidisciplinary Research PartnershipBioinformatics: from nucleotides to networks). The computational resources (STEVIN Supercomputer Infrastructure) and services used in this work were kindly provided by Ghent University, the Flemish Supercomputer Center (VSC), the Hercules Foundation, and the Flemish Governmentdepartment EWI.
[1] A. T. Winfree, Nature 311, 611 (1984).

[2] A. M. Zhabotinsky and A. N. Zaikin, J. Theor. Biol. 40, 45 (1973).

[3] S. Jakubith, H. H. Rotermund, W. Engel, A. von Oertzen, and G. Ertl, Phys. Rev. Lett. 65, 3013 (1990).

[4] K. Agladze, J. P. Keener, S. C. Muller, and A. V. Panfilov, Science 264, 1746 (1994).

[5] G. Gerisch, Naturwissenschaften 58, 430 (1971).

[6] F. Siegert and C. J. Weijer, Proc. Natl. Acad. Sci. USA 89, 6433 (1992).

[7] N. A. Gorelova and J. Bures, J. Neurobiol. 14, 353 (1983).

[8] J. Lechleiter, S. Girard, E. Peraltal, and D. Clapham, Science 252, 123 (1991).

[9] G. Dupont, A. Goldbeter, R. Salomonsz, W. Baxter, and J. Jalife, Bioessays 14, 485 (1992)

[10] M. A. Allessie, F. I. M. Bonke, and F. J. G. Schopman, Circ. Res. 33, 54 (1973).

[11] J. M. Davidenko, P. F. Kent, D. R. Chialvo, D. C. Michaels, and J. Jalife, Proc. Natl. Acad. Sci. USA 87, 8785 (1990).

[12] J. M. Davidenko, A. V. Pertsov, R. Salomonsz, W. Baxter, and J. Jalife, Nature 355, 349 (1992).

[13] R. A. Gray, J. Jalife, A. V. Panfilov, W. T. Baxter, C. Cabo, J. M. Davidenko, and A. M. Pertsov, Science 270, 1222 (1995).

[14] R. A. Gray, J. Jalife, A. V. Panfilov, W. T. Baxter, C. Cabo, J. M. Davidenko, and A. M. Pertsov, Circ. Res. 91, 2454 (1995).
[15] V. G. Fast and A. M. Pertsov, Biofizika. 35, 478 (1990).

[16] J. A. Abildskov and R. L. Lux, J. Cardiovasc. Electrophysiol. 2, 224 (1991).

[17] K. H. ten Tusscher and A. V. Panfilov, Am. J. Physiol. Heart. Circ. Physiol. 284, H542 (2003).

[18] J. A. Abildskov and R. L. Lux, J. Electrocardiol. 33, 55 (2000).

[19] V. I. Krinsky, Biofizika 11, 676 (1966).

[20] A. G. Kleber and Y. Rudy, Physiol. Rev. 84, 431 (2004).

[21] A. V. Panfilov and B. N. Vasiev, Physica D 49, 107 (1991).

[22] Z. Qu, A. Garfinkel, and J. N. Weiss, Biophys. J. 91, 793 (2006).

[23] Z. Qu, A. Garfinkel, and J. N. Weiss, Biophys. J. 91, 805 (2006).

[24] R. R. Aliev and A. V. Panfilov, J. Theor. Biol. 181, 33 (1996).

[25] R. H. Keldermann, K. H. W. J. ten Tusscher, M. P. Nash, R. Hren, P. Taggart, and A. V. Panfilov, Am. J. Physiol. Heart Circ. Physiol. 294, H764 (2008).

[26] R. H. Keldermann, K. H. W. J. ten Tusscher, M. P. Nash, C. P. Bradley, R. Hren, P. Taggart, and A. V. Panfilov, Am. J. Physiol. Heart. Circ. Physiol. 296, H370 (2009).

[27] A. V. Glukhov, V. V. Fedorov, Q. Lou, V. K. Ravikumar, P. W. Kalish, R. B. Schuessler, N. Moazami, and I. R. Efimov, Circ. Res. 106, 981 (2010).

[28] K. H. W. ten Tusscher and A. V. Panfilov, Am. J. Physiol. Heart. Circ. Physiol. 291, H1088 (2006).

[29] J. P. Keener and J. Sneyd, Mathematical Physiology (SpringerVerlag, New York, 1998). 
[30] K. H. W. ten Tusscher, D. Noble, P. J. Noble, and A. V. Panfilov, Am. J. Physiol. Heart Circ. Physiol. 286, H1573 (2004).

[31] S. Rush and H. Larsen, IEEE Trans. Biomed. Eng. 25, 389 (1978).

[32] W. Shimizu and C. Antzelevitch, Circulation 98, 2314 (1998).

[33] W. Shimizu and C. Antzelevitch, J. Am. Coll. Cardiol. 35, 778 (2000).

[34] A. Defauw, I. V. Kazbanov, H. Dierckx, P. Dawyndt, and A. V. Panfilov, PLoS one 8, e79607 (2013).

[35] See Supplemental Material at http://link.aps.org/supplemental/ 10.1103/PhysRevE.88.062703 for movies.

[36] K. J. Sampson and C. S. Henriquez, Am. J. Physiol. Heart Circ. Physiol. 289, H350 (2005).

[37] D. Streeter, Handbook of Physiology, Am Physiol Soc. Chap. Gross Morphology and Fiber Geometry of the Heart (The Heart. Bethesda, Maryland, 1979), Sec. 2. Vol. I, pp. 61-112.
[38] K. F. Wenckebach, Z. Klin. Med. 37, 475 (1899).

[39] A. V. Panfilov and J. P. Keener, Int. J. Bif. Chaos. 3, 445 (1993).

[40] L. Boersma, Z. Zetelaki, J. Brugada, and M. Allessie, Circ. Res. 105, 3053 (2002).

[41] M. Vinson, A. Pertsov, and J. Jalife, Physica D 72, 119 (1994).

[42] T. Wu, M. Yashima, F. Xie, C. A. Athill, Y. Kim, M. C. Fishbein, Z. Qu, A. Garfinkel, J. N. Weiss, H. S. Karagueuzian, and P. Chen, Circ. Res. 83, 448 (1998).

[43] C. W. Zemlin and A. M. Pertsov, Phys. Rev. Lett. 109, 038303 (2012).

[44] T. K. Shajahan, S. Sinha, and R. Pandit, Phys. Rev. E 75, 011929 (2007).

[45] B. O. Bingen, S. F. Askar, M. J. Schalij, I. V. Kazbanov, D. L. Ypey, A. V. Panfilov, and D. A. Pijnappels, Cardiovasc. Res. 97, 161 (2013).

[46] K. Campbell, C. J. Calvo, S. Mironov, T. Herron, O. Berenfeld, and J. Jalife, J. Physiol. 590, 6363 (2012). 